\title{
REPRESENTAÇÃO E PROPAGAÇÃO DE INCERTEZAS EM DADOS DE SOLO. II - ATRIBUTOS NUMÉRICOS ${ }^{(1)}$
}

\author{
S. BÖNISCH ${ }^{(2)}$, M. L. LOPES ASSAD ${ }^{(3)}$, \\ A. M. V. MONTEIRO ${ }^{(4)}$ \& G. CÂMARA ${ }^{(4)}$
}

\begin{abstract}
RESUMO
Este trabalho teve por objetivos utilizar krigagem por indicação para espacializar propriedades de solos expressas por atributos numéricos, gerar uma representação acompanhada de medida espacial de incerteza e modelar a propagação de incerteza por procedimentos fuzzy de álgebra de mapas. Foram estudados os atributos: teores de potássio (K) e de alumínio (Al) trocáveis, saturação por bases (V), soma de bases (S), capacidade de troca catiônica (CTC) e teor de areia total (AT), extraídos de 222 perfis pedológicos e de 219 amostras extras, localizados no estado de Santa Catarina. Quando os atributos foram expressos em classes de fertilidade, a incerteza de $\mathrm{Al}, \mathrm{S}$ e $\mathrm{V}$ aumentou e a de $\mathrm{K}$ e CTC diminuiu, considerando intervalos de confianca de $95 \%$ de probabilidade. Constatou-se que um maior número de dados numéricos de $\mathrm{K}$, S e V levou a uma maior incerteza na inferência espacial, enquanto o maior número de dados numéricos de AT e CTC diminuiu o grau de incerteza. A incerteza diminuiu quando diferentes representações numéricas foram integradas.
\end{abstract}

Termos de indexação: krigagem por indicação, análise espacial, geoestatística,

lógica fuzzy, álgebra de mapa, SIG.

${ }^{(1)}$ Extraído da Tese de Mestrado apresentada, pelo primeiro autor, ao Programa de Pós-Graduação em Sensoriamento Remoto do Instituto Nacional de Pesquisas Espaciais - INPE. Trabalho realizado com Bolsa da Fundação Coordenação de Aperfeiçoamento de Pessoal de Nível Superior. Recebido para publicação em junho de 2002 e aprovado em outubro de 2003.

(2) Mestre em Sensoriamento Remoto, Instituto Nacional de Pesquisas Espaciais - INPE. Av. dos Astronautas 1758, CEP 12227-010 São José dos Campos (SP). E-mail: sbonisch@cwb.matrix.com.br

(3) Professora do DRNPA/CCA, Universidade Federal de São Carlos - UFSCar. CEP 13600-970 Araras (SP). E-mail: assad@cca.ufscar..br

(4) Pesquisador do Instituto Nacional de Pesquisas Espaciais - INPE. CEP 12227-010 São José dos Campos (SP). E-mail: miguel@dpi.inpe.br; gilberto@dti.inpe.br 


\title{
SUMMARY: REPRESENTATION AND PROPAGATION OF SOIL DATA UNCERTAINTIES. II - NUMERIC ATTRIBUTES
}

\begin{abstract}
The objectives of this study were to use kriging indicators to generate a representation of soil properties expressed by numeric attributes, to assess the uncertainty in estimates, and to model the uncertainty propagation generated by the fuzzy procedures of map algebra. The studied attributes were exchangeable potassium (K) and aluminum (Al) contents, sum of bases (SB), cationic exchange capacity (CEC), base saturation (V), and total sand content (TST), extracted from 222 pedologic profiles and 219 extra samples, located in Santa Catarina State, Brazil. When the attributes were expressed in fertility classes, the uncertainty of $A l, S B$, and $V$ increased while the uncertainty of $K$ and $C E C$ decreased, for intervals of confidence of $95 \%$ probability. A larger number of numeric data for $K, S B$, and $V$ provided a larger uncertainty for space inference, while the uncertainty degree decreased for the largest number of numeric data of TS and CEC. There was a decrease of uncertainty when different numeric representations were integrated by fuzzy procedures of map algebra.
\end{abstract}

Index terms: kriging indicator, spatial analysis, geostatistics, fuzzy logic, map algebra, GIS.

\section{INTRODUÇÃO}

McBratney et al. (2000) destacam que os métodos convencionais de levantamentos de solos foram criticados no passado - talvez justificadamente - por serem muito qualitativos nas caracterizações. Nos últimos anos, observa-se o desenvolvimento de modelos quantitativos, categorizados em um novo ramo da ciência do solo conhecido como Pedometria (McBratney et al., 2000), formalmente definida por Webster (1994) como um neologismo derivado dos termos de origem grega pedon (solo) e metron (medida). Países, como Austrália, Estados Unidos, França e Holanda, dentre outros, têm mudado a abordagem tradicional de estudo dos recursos naturais, adotando modelos quantitativos, técnicas de predição espacial, determinação de precisão e de incertezas de atributos e integração de dados em sistema de informações geográficas (SIGs).

O trabalho em SIG pode ser dividido em cinco etapas (Bonham-Carter, 1990): construção de banco de dados; extração das evidências relevantes; construção de modelos; visualização dos dados de saída e interpretação dos resultados. Conforme destaca Barbosa (1997), uma das características importantes dos SIGs é representar a informação espacial numericamente modelando-a por estatística espacial (geoestatística) e por álgebra de mapas. A álgebra de mapas ou álgebra de campos pode ser vista como uma extensão da álgebra tradicional e constitui um conjunto de operadores onde as variáveis manipuladas correspondem a geocampos (Berry, 1993).

As técnicas quantitativas de predição espacial em levantamentos de solos são geralmente derivadas da geoestatística e de métodos estatísticos modernos (McBratney et al., 2000). Dentre os métodos geoestatísticos disponíveis, cita-se a krigagem na qual a determinação da distribuição de probabilidade associada ao atributo é não-paramétrica (Goovaerts, 1997). No tratamento de atributos numéricos, estima-se um conjunto de valores que representam uma aproximação discretizada do modelo de distribuição de probabilidade de valores do atributo. Na krigagem por indicação os valores numéricos são modificados por uma codificação por indicação, ou seja, faz-se uma transformação não-linear, que, para um conjunto amostral $\mathrm{Z}\left(\mathrm{u}=\mathrm{u}_{\alpha}\right)$, dado um valor de corte $z_{k}$, gera um conjunto amostral por indicação $\mathrm{I}\left(\mathrm{u}=\mathrm{u} \alpha ; z_{k}\right)$, do tipo:

$$
\begin{aligned}
& \mathrm{I}\left(\mathrm{u} ; z_{k}\right)=1, \text { se } \mathrm{Z}(\mathrm{u}) \leq z_{k} \\
& \mathrm{I}\left(\mathrm{u} ; z_{k}\right)=0, \text { se } \mathrm{z}(\mathrm{u})>z_{k} .
\end{aligned}
$$

O conjunto amostral por indicação é usado para inferir os valores do atributo nas posições não amostradas e essa inferência é feita por meio da esperança condicional da variável aleatória numérica por indicação, dada por:

$$
\begin{gathered}
\mathrm{E}\left\{\mathrm{I}\left(\mathrm{u} ; z_{k}\right) \mid(n)\right\}=1 * \operatorname{Prob}\left\{\mathrm{I}\left(\mathrm{u} ; z_{k}\right)=1 \mid(n)\right\}+ \\
0 * \operatorname{Prob}\left\{\mathrm{I}\left(\mathrm{u} ; z_{k}\right)=0 \mid(n)\right\}= \\
\left.1 * \operatorname{Prob}\left\{\mathrm{I}\left(\mathrm{u} ; z_{k}\right)=1 \mid(n)\right\}=\mathrm{F}^{*}\left(\mathrm{u} ; z_{k}\right) \mid(n)\right)
\end{gathered}
$$

A esperança condicional de $\mathrm{I}\left(\mathrm{u} ; z_{k}\right)$ fornece, para o valor de corte $\mathrm{z}=z_{k}$, uma estimativa de $\mathrm{F}^{*}(\mathrm{u}$; $\left.z_{k} \mid(n)\right)$, que é a função de distribuição de probabilidade acumulada condicionada (fdpac) para atributos numéricos, e pode ser estimada por algoritmos de krigagem, como a krigagem ordinária. Desta forma, podem ser estimados valores da fdpac de Z $(u)$ para vários valores de corte, possibilitando a construção de uma aproximação discretizada da fdpac de Z(u). Quanto maior o conjunto de valores de corte, melhor será esta aproximação. 
Alguns valores característicos da distribuição de probabilidade, como valor médio e variância, podem ser estimados diretamente dos valores discretizados. O valor médio esperado $E(Z(u))$ de uma variável aleatória pode ser estimado por:

$$
\mathrm{E}[\mathrm{Z}(\mathrm{u})]=\int_{-\infty}^{\infty} \mathrm{z} \cdot \mathrm{f}(\mathrm{u} ; \mathrm{zl}(\mathrm{n})) \delta \mathrm{z}=\int_{-\infty}^{\infty} \mathrm{z} \cdot \delta \mathrm{F}(\mathrm{u} ; \mathrm{zl}(\mathrm{n}))
$$

E, a partir de $K$ valores de corte, $\mu_{\mathrm{z}}(\mathrm{u})$ pode ser estimado pela aproximação:

$$
\mu_{\mathrm{Z}}(\mathrm{u})=\int_{-\infty}^{\infty} \mathrm{z} . \delta \mathrm{F}(\mathrm{uzl}(\mathrm{n}))=\sum_{\mathrm{k}=1}^{\mathrm{K}+\mathrm{l}} \mathrm{Z}_{\mathrm{k}}\left[\mathrm{F}^{*}\left(\mathrm{u} ; \mathrm{Z}_{\mathrm{k}} \mathrm{I}(\mathrm{n})\right) \mathrm{F}^{*}\left(\mathrm{u} ; \mathrm{Z}_{\mathrm{k}-\mathrm{l}} \mathrm{I}(\mathrm{n})\right)\right]
$$

em que os valores das $\mathrm{F}^{*}\left(\mathrm{u} ; z_{k} \mid(n)\right), k=1,2, \ldots, K$, são os valores estimados das fdpac's para cada valor $z_{k}$ do atributo, $z_{o}=z_{\min }, z_{k+1}=z_{\max }, z^{\prime}{ }_{k}=\left(z_{k}+z_{k-1}\right) / 2$, $\mathrm{F}\left(\mathrm{u} ; z_{o} \mid(n)\right)=0$ e $\mathrm{F}\left(\mathrm{u} ; z_{k+1} \mid(n)\right)=1$.

Da mesma forma, a variância $\left(\sigma^{2}\right)^{*}(u)$ pode ser estimada por:

$$
\left(\sigma^{2}\right)^{*}(\mathrm{u})=\int_{-\infty}^{\infty}\left[\mathrm{z}-\mu_{\mathrm{z}}(\mathrm{u})\right]_{\delta}^{\mathrm{F}} \mathrm{F}(\mathrm{u} ; \mathrm{z} \mid(\mathrm{n})) \approx \sum_{\mathrm{k}=1}^{\mathrm{K}+1}\left[\mathrm{z}_{\mathrm{k}}^{\prime}-\mu_{\mathrm{z}}(\mathrm{u})\right]^{[}\left[\mathrm{F}^{*}\left(\mathrm{u} ; \mathrm{z}_{\mathrm{k}} \mid(\mathrm{n})\right)-\mathrm{F}^{*}\left(\mathrm{u} ; \mathrm{z}_{\mathrm{k}-1} \mid(\mathrm{n})\right)\right]
$$

A partir destas métricas, podem ser estimados intervalos de confiança, que são a forma mais comum de expressar incertezas, ou seja, o intervalo de confiança indica a probabilidade de um valor desconhecido estar entre um valor mínimo e um valor máximo. Entretanto, há duas suposições que devem ser aceitas para o uso de intervalos de confiança (Isaaks \& Srivastava, 1989): (a) a fdpac deve ser próxima de uma distribuição normal e (b) o valor estimado para a variância deve ser um valor acurado.

A propagação de incertezas em SIG corresponde ao tratamento das incertezas associadas aos dados de entrada, quando estes são submetidos a operações de álgebra de mapas. Segundo Tomlin (1990), as operações de álgebra de mapas sobre geocampos podem ser classificadas como pontuais, zonais e de vizinhança. Quando as representações de entrada são referentes a atributos numéricos, essas operações correspondem a operações locais pontuais e as incertezas são expressas de acordo com a variância dos atributos numéricos.

Dentre os métodos de propagação de incertezas (Heuvelink, 1998), o método de Taylor de primeira ordem consiste de uma expansão das séries de Taylor de primeira ordem centrada no vetor b. Este vetor b é um vetor de valores médios de $N$ variáveis aleatórias $\mathrm{Z}_{\mathrm{i}}, \mathrm{i}=1, \ldots, N$ e pode ser indicado por:

$$
\mathrm{b}=\left(\mu_{\mathrm{z} 1}, \ldots, \mu_{\mathrm{zn}}\right)
$$

A expansão da série de Taylor de primeira ordem em torno de $b$ pode ser escrita como:

$$
\mathrm{Y}=\mathrm{g}(\mathrm{Z})=\mathrm{g}(\mathrm{b})+\sum_{\mathrm{i}=1}^{\mathrm{n}}\left[\left(\mathrm{Z}_{\mathrm{i}}-\mathrm{b}_{\mathrm{i}}\right) \cdot\left(\delta \mathrm{g} / \delta \mathrm{z}_{\mathrm{i}} \cdot(\mathrm{b})\right)\right]+\text { resíduo }
$$

Desconsiderando o termo de resíduo, o valor médio $\mathrm{m}$ e a variância $\mathrm{s}^{2}$ de $\mathrm{Y}$ são obtidos por:

$$
\begin{aligned}
& \boldsymbol{\mu}=E[Y]=E\left[g(\mathbf{b})+\sum_{i=1}^{n}\left\{\left(Z_{i}-b_{i}\right)\left(\frac{\delta g}{\delta_{i}}(\mathbf{b})\right)\right\}\right]=g(\mathbf{b}) \\
& \sigma^{2}=E\left[(Y-E[Y])^{2}\right]=E\left[\left(g(\mathbf{b})+\sum_{i=1}^{n}\left\{\left(Z_{i}-b_{i}\right)\left(\frac{\partial g}{\partial z_{i}}(\mathbf{b})\right)\right\}-g(\mathbf{b})\right)^{2}\right] \\
&=E\left[\left(\sum_{i=1}^{n}\left\{\left(Z_{i}-b_{i}\right)\left(\frac{\delta g}{\delta z_{i}}(\mathbf{b})\right)\right\}\left(\sum_{j=1}^{n}\left\{\left(Z_{j}-b_{j}\right)\left(\frac{\delta g}{\delta z_{j}}(\mathbf{b})\right)\right\}\right]\right.\right. \\
&=\sum_{i=1}^{n}\left\{\sum_{j=1}^{n}\left\{\tau_{i j} \sigma_{i} \sigma_{j} \frac{\delta g}{\delta z_{i}}(\mathbf{b}) \cdot \frac{\delta g}{\delta z_{j}}(\mathbf{b})\right\}\right\}
\end{aligned}
$$

Partindo destas equações, verifica-se que $\mathrm{Y}$, a representação de saída, depende apenas das representações $b_{i}$ e que a variância de $Y$, além de depender das correlações e desvios-padrões das variáveis aleatórias $Z_{i}$, depende, também, das primeiras derivadas de $\mathrm{g}(\mathrm{u})$. Isto impõe o uso de operações que admitam o cálculo da $1^{\text {a }}$ derivada, para que esse método de propagação possa ser aplicado.

Existem vários tipos de operadores para integração de dados numéricos (booleano, média ponderada, lógica fuzzy, Bayes, redes neurais artificiais, dentre outros) que geram resultados (geocampos derivados) com representação numérica. A lógica fuzzy, ou lógica nebulosa (fuzzy logic), tem por objetivo modelar, de modo aproximado, o raciocínio humano, visando manipular informações em um ambiente de incerteza e imprecisão, fornecendo uma resposta aproximada para uma questão baseada em um conhecimento que é inexato, incompleto ou não totalmente confiável. Os conjuntos fuzzy não empregam valores de verdade; expressam valores lingüísticos; usam variáveis lingüísticas; e suportam modos de raciocínio aproximado. Um elemento pode pertencer com um certo grau, denominado grau de pertinência a um determinado conjunto fuzzy. $\mathrm{O}$ elemento de um conjunto fuzzy é representado por $\mathrm{MF}_{\mathrm{A}}(z)_{\mathrm{i}} / z_{\mathrm{i}}$, que denota que o elemento $z_{\text {i pertence }}$ ao conjunto fuzzy com grau $\mathrm{MF}_{\mathrm{A}}{ }_{\mathrm{A}}(z)_{\mathrm{i}}$.

A integração por meio de modelagem fuzzy tem sido extensamente utilizada, pois se baseia no conceito de conjunto nebuloso (fuzzy sets), que é uma forma de caracterização de classes, que, por várias razões, não têm, ou não podem definir, limites rígidos (contatos) entre classes (Burrough \& McDonnell, 1998). Esse tipo de modelagem é indicado para lidar com ambigüidades, abstrações e ambivalências em modelos matemáticos complexos que representam contatos difusos, comuns em processos naturais.

Matematicamente, um conjunto nebuloso é definido como:

$$
\mathrm{A}=\left(\mathrm{z}, \mathrm{MF}_{\mathrm{A}}(z)\right) \text { para todo } z \in \mathrm{Z},
$$

ou seja, $Z$ denota um espaço finito de $n$ objetos, e o conjunto nebuloso $A$ em $Z ; M F_{A}(z)$ é a função de 
associação conhecida como grade de associação de $z$ em A, isto é, uma "graduação" mapeável do membro $z$ em $A$ (Burrough \& McDonnell, 1998). Normalmente, o valor de $M F_{A}(z)$ é um número que varia de 0 a 1 , sendo que 1 representa o membro que se encaixa completamente no conjunto e 0 o membro que não pertence ao conjunto (Figura 1). Os graus de pertinência de $z$ ser membro de um conjunto $A$ refletem uma ordem, não baseada em probabilidade, mas na possibilidade de $z$ pertencer a $A$ (Fucks, 1988). O valor da função $M F_{A}(z)$ de um objeto $z$ em $A$ pode ser interpretado como um grau de compatibilidade de um predicado associado ao conjunto $A$ e ao objeto $z$. Assim, $M F_{A}(z)$ avalia o quanto $z$ pode ser pertencente ao conjunto $A$ (Burrough \& McDonnell, 1998).

A função que define conjuntos fuzzy pode ser linear, não-linear, normal, gaussiana, ascendente, descendente, sigmóide, entre outras, sendo as mais utilizadas na determinação de valores de membros fuzzy as funções lineares e quadráticas (Burrough \& McDonnell, 1998). A função linear fuzzy (Figura 1) é definida por dois segmentos de reta inclinados que se encontram em um ponto central de valor $\mathrm{MF}_{\mathrm{A}}(\mathrm{z})$ $=0,5$ e a inclinação das retas define a zona de transição fuzzy. O conjunto fuzzy definido pela função linear é expresso por:

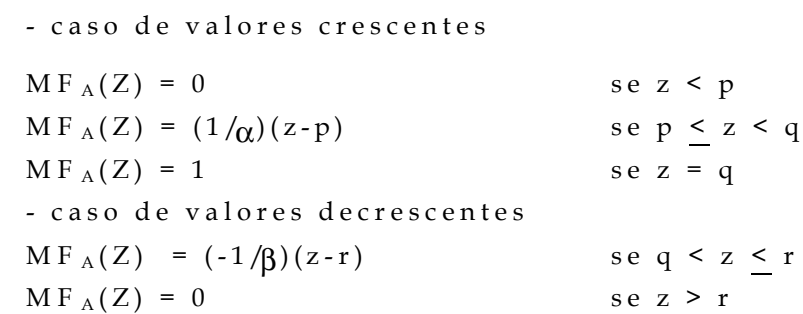

Operações: $A \cup B, A \cap B, \neg A, \neg B$ Fronteiras bem definidas e inflexíveis.

Conjuntos Booleanos

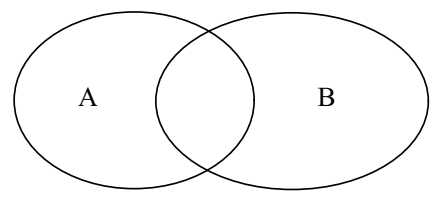

Conjuntos Fuzzy

$\mathrm{MF}_{\mathrm{A}}(\mathrm{z})$ avalia o quanto $\mathrm{z}$ pode ser pertencente ao conjunto A. $\mathrm{MF}_{\mathrm{B}}(\mathrm{z})$ avalia o quanto $\mathrm{z}$ pode ser pertencente ao conjunto $\mathrm{B}$. Por exemplo:

seja: $A=\{$ solos com teor alto do elemento y $\}$ e

$\mathrm{B}=\{$ solos com teor baixo do elemento $\mathrm{y}\}$

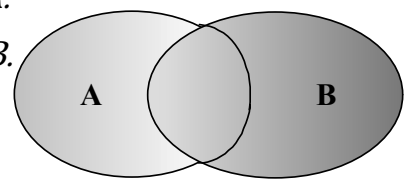

O gráfico mostra as funções lineares de associação (MF) para conjuntos fuzzy "solos com teor alto" e "solos com teor baixo" do elemento y.

teor alto $\geq 8\left(\mathrm{MF}_{\mathrm{A}}(\mathrm{z})=1\right.$ "verdadeiro" e $\mathrm{MF}_{\mathrm{B}}(\mathrm{z})=0$ "falso")

teor baixo $\leq 4\left(\mathrm{MF}_{\mathrm{B}}(\mathrm{z})=1\right.$ "verdadeiro" e $\mathrm{MF}_{\mathrm{A}}(\mathrm{z})=0$ "falso")

A inclinação das retas define a zona de transição fuzzy para os conjuntos A e B.

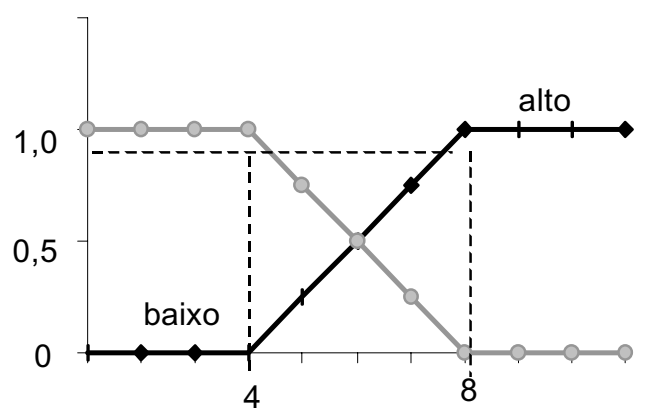

$\mathrm{MF}(\mathrm{A})->$ teor alto $-0-\mathrm{MF}(\mathrm{B})->$ teor baixo

Figura 1. Comparação entre conjuntos booleanos e conjuntos fuzzy (adaptado de Burrough \& McDonnell, 1998). 
em que $\alpha=q-p, \beta=r-q$ e $q$, p e r são valores dos limites de classe de dois conjuntos fuzzy $A$ e $B$.

Este trabalho teve por objetivos espacializar propriedades de solos expressas por atributos numéricos, utilizando krigagem por indicação; gerar, para cada atributo de solo estudado, uma representação acompanhada de medida espacial de incerteza e modelar a propagação de incerteza gerada em procedimentos fuzzy de álgebra de mapas de atributos numéricos. Todo o procedimento se apoiou em duas premissas: (a) os dados de solos utilizados representavam o universo de amostras disponíveis e possíveis de ser manipuladas, não tendo sido objeto deste trabalho adquirir novos dados, e (b) os dados de solos utilizados no trabalho são confiáveis e não foram submetidos à crítica.

\section{MATERIAL E MÉTODOS}

Foram utilizados atributos de solos que serviram de base para o zoneamento pedoclimático para a cultura de soja no estado de Santa Catarina, o qual tem por referência o Projeto de Zoneamento Pedoclimático do Brasil (EMBRAPA, 1997). Foram utilizados dados extraídos de 222 perfis pedológicos e 219 amostras extras (Bönisch et al., 2004), obtidos em diferentes fontes (UFSM, 1972; IBGE, 1986, 1999; Almeida, 1988; Bognola, 1995; Almeida et al., 1997; EMBRAPA, 1997, 1998; Gmach, 1999; Corrêa, 2001).

Foram consideradas as seguintes variáveis: teor de potássio trocável $(\mathrm{K})$, teor de alumínio trocável (Al), saturação por bases (V), soma de bases (S), capacidade de troca catiônica (CTC) e teor de areia total (AT). Todas foram determinadas para cada horizonte, tanto nos perfis quanto nas amostras extras. Nas amostras extras, foram considerados apenas os dados em que a amostra atingiu uma profundidade mínima de $20 \mathrm{~cm}$, já que a concentração das raízes até esta profundidade é de aproximadamente 90 \% (Miyasaka \& Medina, 1981). As amostras que não atingiram esta profundidade mínima foram desconsideradas. Além disso, algumas vezes, dependendo da variável que estava sendo considerada, alguns perfis e amostras não dispunham das informações necessárias e, então, não fizeram parte do respectivo conjunto de amostras.

Visando obter um único valor de cada propriedade por ponto do espaço, foi aplicada uma média ponderada aos dados disponíveis para horizontes, em que o peso atribuído dependeu da percentagem de raízes de soja normalmente presente na profundidade correspondente àquele horizonte, conforme indicado em Bönisch et al. (2004).

Os procedimentos de espacialização foram precedidos pela análise exploratória dos dados (módulo geoestatística do SPRING®), por meio de estatísticas univariadas que compreenderam medidas de posição (média, valores mínimo e máximo, quartis inferior e superior e mediana) e medidas de dispersão (variância, desvio-padrão e coeficientes de assimetria, de curtose e de variação). Foram feitos também histogramas dos valores dos atributos e o gráfico de probabilidade normal.

O primeiro passo na espacialização dos atributos numéricos considerados neste estudo foi a codificação por indicação, para a geração dos conjuntos amostrais por indicação. Na codificação, para cada uma das propriedades foram determinados nove valores de corte, definidos pelo critério de igualdade do número de amostras em cada corte. A análise de variografia foi feita para cada conjunto amostral, visando à determinação dos parâmetros dos modelos de semivariogramas teóricos ajustados aos semivariogramas experimentais.

Em seguida, foi feita a inferência dos valores de probabilidade acumulada nas posições não amostradas, usando o interpolador de krigagem ordinária. Esta inferência foi feita para cada conjunto amostral, de cada atributo estudado.

Uma variável aleatória contínua $[\mathrm{Z}(\mathrm{u})]$, como as analisadas neste trabalho, pode assumir valores contínuos no espaço segundo uma ordem natural (relação de ordem). Um cuidado que se deve ter com os resultados obtidos nos procedimentos de krigagem por indicação é quanto aos desvios de relação de ordem (Goovaerts, 1997). Eles ocorrem porque: (a) não são usados os mesmos pesos de estimativa da krigagem para todos os valores de corte e (b) porque a krigagem não garante que todos os pesos sejam positivos. Com atributos numéricos, podem ocorrer dois problemas com os valores inferidos de probabilidade acumulada e que resultam em desvios de relação de ordem. O primeiro é que podem ser inferidos valores menores que 0 e maiores que 1 ; o segundo é que o valor estimado para um valor de corte $z_{j}$ pode vir a ser maior que o valor estimado para um valor de corte $z_{k}$, quando $z_{j} \leq z_{k}$ (Felgueiras, 1999). A solução adotada para o primeiro problema foi ajustar os valores estimados para estes limites, mapeando os valores negativos para 0 e os valores maiores que 1 para 1 .

A solução do segundo problema foi construir duas fdpac - máxima e mínima - de acordo com a função da fdpac estimada; em seguida, fez-se a média entre elas, conforme proposto por Deutsch \& Journel (1998) (Figura 2). Na construção da fdpac máxima, partiu-se do valor estimado para o primeiro corte $z_{k}$ e o valor do corte seguinte $\left(z_{k}+1\right)$ devia ser igual ou superior ao valor do corte $z_{k}$. Quando o valor do corte $z_{k+1}$ foi menor, este foi ignorado e ao corte $z_{k}+1$ foi atribuído o valor correspondente ao corte $z_{k}$. Na construção da fdpac mínima, adotou-se a regra inversa e partiu-se do valor do último corte, para que o valor do corte anterior $\left(z_{k-1}\right)$ fosse inferior ou igual ao valor do corte em questão. Quando o valor do corte $z_{k-1}$ foi maior, este foi ignorado e ao corte $z_{k-1}$ 
foi atribuído o valor correspondente ao corte $z_{k}$ (Deutsch \& Journel, 1998).

Partindo dos resultados corrigidos, foram estimados os valores médios e as variâncias de cada uma das variáveis. A espacialização dos atributos estudados e suas incertezas associadas (Figuras 3 e 4) foram expressas em intervalos de confiança de $95 \%$ de probabilidade, ou seja, considerou-se um intervalo de $95 \%$ de probabilidade do valor estimado para a média m estar entre os valoresn $\mu \pm 2$ s, sendo s o desvio-padrão.

Em seguida, visando testar o efeito de funções de álgebra de mapas na propagação de incertezas associadas às representações numéricas, os atributos numéricos $\mathrm{K}$, Al, S, CTC e V foram integrados para espacialização da limitação das terras em fertilidade. Nesta etapa, foi necessário, inicialmente, expressar cada atributo de acordo com a aptidão em fertilidade (Quadro 1).

O primeiro passo consistiu na classificação contínua das representações desses atributos, utilizando o mapeamento fuzzy. A função de associação escolhida foi uma função linear, com os valores dos parâmetros $p$ e $q$ definidos em função

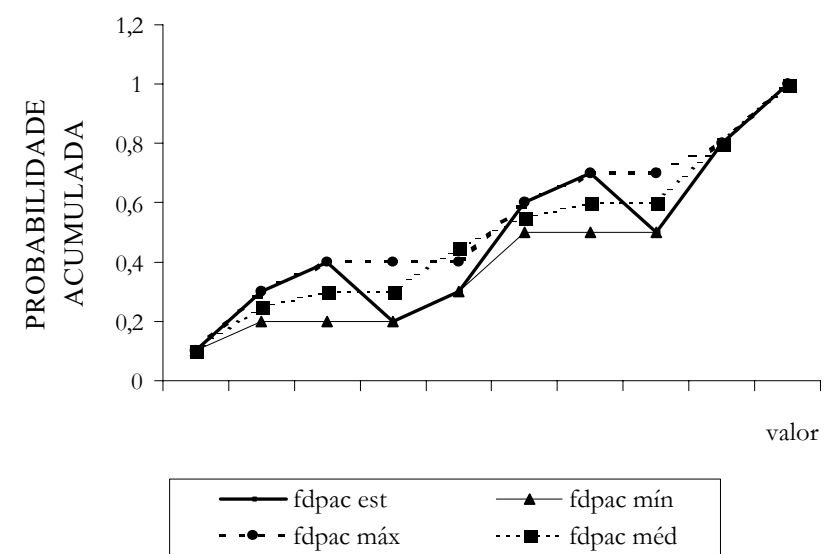

Figura 2. Representação da correção do desvio de relação de ordem na modelagem de atributos numéricos, utilizando krigagem por indicação (adaptado de Deutsch \& Journel, 1998). dos valores dos limites dos intervalos dos atributos químicos, que foram relacionados com uma fertilidade regular $(p)$ e com uma fertilidade boa $(q)$, que corresponderam às classes de aptidão boa e regular, respectivamente (Quadro 2). Isto porque, no Zoneamento Pedoclimático do Brasil para a cultura da soja de Santa Catarina (EMBRAPA, 1997), considerou-se o cultivo no nível de manejo $C$, ou seja, com uso de corretivos e fertilizantes. Neste caso, as classes de limitação em fertilidade alta, média e baixa (Quadro 1) conferem aptidão boa (fertilidade boa) e a classe de fertilidade muito baixa confere uma aptidão regular (fertilidade regular).

Quadro 2. Funções lineares fuzzy utilizadas na classificação contínua das representações dos atributos numéricos: teor de potássio $(K)$, teor de alumínio trocável (Al), soma de bases (S), capacidade de troca catiônica (CTC), saturação por bases ( $V$ ), expressos de acordo com a aptidão em fertilidade

K (mg kg-1)

$$
\begin{aligned}
& M F_{K}(Z)=0 \\
& M F_{K}(Z)=(z-45) / 35 \\
& M F_{K}(Z)=1 \\
& \text { Al (c mol } \left.\mathrm{kg}^{-1}\right) \\
& \mathrm{MF}_{\mathrm{A} 1}(\mathrm{Z})=1 \\
& \mathrm{MF}_{\mathrm{Al}}(\mathrm{Z})=(4-\mathrm{z}) / 3,7 \\
& \mathrm{MF}_{\mathrm{A} 1}(\mathrm{Z})=0 \\
& \mathrm{~S}\left(\mathrm{cmol}_{\mathrm{c}} \mathrm{kg}^{-1}\right) \\
& M F_{s}(Z)=0 \\
& \operatorname{MFs}_{\mathrm{S}}(Z)=(\mathrm{z}-2) / 2 \\
& M F_{S}(Z)=1 \\
& \text { CTC }\left(\mathrm{cmol}_{\mathrm{c}} \mathrm{kg}^{-1}\right) \\
& \mathrm{MF}_{\text {Стс }}(Z)=0 \\
& \operatorname{MF}_{\text {с т с }}(Z)=(z-10) / 40 \\
& \operatorname{MF}_{\text {стс }}(Z)=1 \\
& \text { se } z<45 \\
& \text { se } 45 \leq z<80 \\
& \text { se } z>-80 \\
& \text { se } z \leq 0,3 \\
& \text { se } 0, \overline{3}<z \leq 4 \\
& \text { se } z>4 \\
& \text { se } z<2 \\
& \text { se } 2<z<4 \\
& \text { se } z \geq 4 \\
& \text { se } z<10 \\
& \text { se } 10<z<50 \\
& \text { se } z>\overline{5} 0
\end{aligned}
$$

\begin{tabular}{|c|c|c|c|c|}
\hline Classe de fertilidade(2) & Alta & Média & Baixa & Muito baixa \\
\hline $\mathrm{K}, \mathrm{mg} \mathrm{kg}^{-1}$ & $\geq 80$ & $\geq 45$ e $<80$ & $<45$ & $<45$ \\
\hline $\mathrm{Al}, \mathrm{cmol}_{\mathrm{c}} \mathrm{kg}^{-1}$ & $<0,3$ & $\geq 0,4$ e $<1$ & $\geq 1 \mathrm{e}<4$ & $\geq 4$ \\
\hline $\mathrm{S}, \mathrm{cmol}_{\mathrm{c}} \mathrm{kg}^{-1}$ & $\geq 4$ & $\geq 2$ e $<4$ & $<2$ & $<2$ \\
\hline $\mathrm{CTC}, \mathrm{cmol}_{\mathrm{c}} \mathrm{kg}^{-1}$ & $\geq 50$ & $\geq 35$ e $<50$ & $\geq 10$ e $<35$ & $<10$ \\
\hline $\mathrm{V}(\%)$ & $\geq 8$ & $\geq 6$ e $<8$ & $\geq 4 \mathrm{e}<6$ & $<4$ \\
\hline
\end{tabular}

Quadro 1. Critérios para classificação dos atributos solos em classes de fertilidade ${ }^{(1)}$ das variáveis: teor de potássio (K), teor de alumínio trocável (Al), soma de bases (S), capacidade de troca catiônica (CTC), saturação por bases (V), tomando por base as exigências da cultura de soja

\footnotetext{
${ }^{(1)}$ Fonte: CEPA (1985), citado por Embrapa (1997), e Ramalho Filho \& Beek (1995). ${ }^{(2)}$ Adaptado de Embrapa (1997).
} 
No caso do teor de K, por exemplo, primeiramente foi feito um mapeamento para os limites do intervalo, isto é, todos os valores maiores que $80 \mathrm{mg} \mathrm{kg}^{-1}$ foram mapeados para $80 \mathrm{mg} \mathrm{kg}^{-1}$ e todos os valores menores que $45 \mathrm{mg} \mathrm{kg}^{-1}$ foram mapeados para $45 \mathrm{mg} \mathrm{kg}{ }^{-1}$. Em seguida, para valores de teor de $\mathrm{K}$ iguais a $45 \mathrm{mg} \mathrm{kg}^{-1}$, atribuiu-se o valor 0 , e, para valores iguais a $80 \mathrm{mg} \mathrm{kg}^{-1}$, atribuiu-se valor 1 . Aos valores entre 45 e $80 \mathrm{mg} \mathrm{kg}^{-1}$, foram atribuídos valores entre 0 e 1 , segundo função linear fuzzy.

$\mathrm{O}$ efeito de funções de álgebra de mapas na propagação de incertezas associadas às representações numéricas pôde ser avaliado na integração das representações dos atributos $\mathrm{K}, \mathrm{Al}, \mathrm{S}, \mathrm{CTC}$ e V por meio do operador fuzzy soma ponderada. A propagação das incertezas, neste procedimento de classificação fuzzy, foi efetuada pelo método de Taylor de primeira ordem, aplicado em duas etapas. A primeira considerou as operações envolvidas no mapeamento dos valores fora do intervalo considerado (por exemplo, [45-80] para o caso do teor de K) para os limites deste intervalo. Estas operações consistiram na adição ou subtração de constantes, cujas derivadas foram nulas e, neste caso, os valores de variância propagados por Taylor de primeira ordem são exatamente os mesmos valores das variâncias do atributo considerado. $\mathrm{Na}$ segunda etapa, considerou-se a função linear do mapeamento fuzzy aplicada para todos os valores do atributo, mapeados para os limites do intervalo considerado, ou não, gerando, como resultados, as representações da variância de cada atributo.

$\mathrm{Na}$ integração das representações de cada atributo estudado de acordo com a aptidão em fertilidade, foi aplicado um operador fuzzy soma ponderada, em que o somatório dos pesos era igual a 1. A equação deste operador, conforme Burrough \& McDonnell (1998), é:

$$
M F_{A}=\sum_{j=1}^{k} w_{j} M F_{A j} \text { em que } \sum_{j=1}^{k} w_{j}=1, w_{j}>0
$$

Foi atribuído peso igual a 0,2 para todas as representações, isto é, considerou-se que os cinco atributos (K, Al, S, CTC e V) contribuíam, de forma eqüitativa, na estimativa da limitação das terras em fertilidade. Isto fez com que a operação fuzzy se tornasse uma média simples das representações. A propagação das variâncias foi feita pelo método de Taylor de primeira ordem.

Todos os procedimentos de espacialização usando krigagem por indicação, com ajustes de semivariogramas, propagação de incertezas e mapeamento fuzzy foram feitos no SPRING® versão 3.4, desenvolvido pelo INPE - Instituto Nacional de Pesquisas Espaciais.

\section{RESULTADOS E DISCUSSÃO}

A estatística dos dados de perfis e de amostras de solos efetivamente utilizados para a predição espacial (Quadro 3) revela que os valores médios dos atributos de solos no estado de Santa Catarina indicaram características de baixa fertilidade e textura média. Os parâmetros de localização evidenciaram a grande variedade de solos, com valores máximos e mínimos apontando para a existência de solos de textura e fertilidade contrastantes. As medidas de dispersão (variância e desvio-padrão) confirmaram a amplitude dos solos utilizados neste estudo. Todos os atributos estudados apresentaram coeficientes de variação (CV) bastante elevados, indicando que os perfis e amostras considerados recobriam uma ampla gama de valores. Vauclin (1983), a partir de uma compilação não exaustiva de resultados publicados entre 1972 e 1982, classificou variáveis e propriedades físico-químicas de solos em três grupos (pequena, média e forte variabilidade), considerando os valores de CV obtidos, e constatou que as propriedades químicas apresentavam variabilidade nitidamente maior de um ponto a outro de uma parcela de estudo do que as propriedades físicas.

No presente estudo, feito em escala regional, como era de se esperar, os CV foram ainda mais elevados do que em Vauclin (1983). No teor de potássio, por exemplo, Vauclin (1983) aponta CV da ordem de 37, 56 e $89 \%$, para médias de 847, 560 e $672 \mathrm{mg} \mathrm{kg}^{-1}$, respectivamente, enquanto para o teor de areia total o maior CV constatado foi de $42 \%$ para um valor médio de $260 \mathrm{~g} \mathrm{~kg}^{-1}$.

Em todos os atributos estudados, a distribuição foi positivamente assimétrica (histograma levemente alongado à direita), tendo sido a assimetria maior para os atributos S, CTC e K (Quadro 3). Quanto ao grau de achatamento, os coeficientes de curtose obtidos indicaram que CTC, S, e K apresentavam distribuição positivamente achatada, enquanto a $\mathrm{Al}$ e AT foram ligeiramente platicúrticas; V apresentou distribuição leptocúrtica (negativamente achatada). A média e a mediana apresentaram valores próximos para CTC, indicando distribuição aproximadamente simétrica. Nas demais variáveis, as diferenças entre média e mediana foram mais elevadas, indicando distribuição assimétrica à direita.

Os atributos $\mathrm{K}$ e AT foram ajustados por semivariogramas unidirecionais em todos os intervalos de corte considerados, indicando que para esses a variabilidade espacial foi isotrópica. Por outro lado, após análise das diferentes configurações dos dados de CTC, S, V e Al, constatou-se que em pelo menos um dos intervalos de corte (Quadros $4 \mathrm{e}$ 5), o ajuste foi obtido com dois semivariogramas, indicando anisotropia dos dados: alguns associados à direção Norte (próximos de $0^{\circ}$ ) e outros associados à direção Leste (próximos de $90^{\circ}$ ). Ressalta-se que a anisotropia ocorreu basicamente nos valores intermediários desses atributos $\left(3^{\circ}, 4^{\circ}\right.$ e $5^{\circ}$ corte $)$. Isto significa que, em solos com valores de CTC, S, $\mathrm{V}$ e Al muito baixos ou muito altos, a direção assumida na amostragem dos solos não interfere na 
Quadro 3. Sumário da estatística dos dados de solos utilizados

\begin{tabular}{|c|c|c|c|c|c|c|}
\hline & $\mathbf{A T}{ }^{(1)}$ & K troc. & Altroc. & S & C TC & V \\
\hline & $\mathrm{g} \mathrm{kg}^{-1}$ & $\mathrm{mg} \mathrm{kg}^{-1}$ & 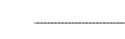 & $\mathrm{mol}_{\mathrm{c}} \mathrm{kg}$ & - & $\%$ \\
\hline$N^{(2)}$ & 353 & 349 & 351 & 350 & 350 & 350 \\
\hline Média & 21,85 & 91,22 & 3,38 & 4,46 & 16,48 & 27,02 \\
\hline Mediana & 15,1 & 72 & 2,9 & 2,15 & 15,57 & 16,9 \\
\hline Máximo & 91,8 & 522 & 14,2 & 42,93 & 79,61 & 95,27 \\
\hline Mínimo & 0 & 8 & 0 & 0,3 & 1,23 & 0,96 \\
\hline Variância & 341,36 & 5475,51 & 7,76 & 32,85 & 71,03 & 608,56 \\
\hline Quartil inferior & 7 & 43 & 1,05 & 1,2 & 11,46 & 7,64 \\
\hline Quartil superior & 33,2 & 109 & 5,2 & 4,94 & 20,05 & 40,41 \\
\hline Desvio-padrão & 18,48 & 74 & 2,79 & 5,73 & 8,43 & 24,67 \\
\hline Coeficiente variação & 0,85 & 0,81 & 0,83 & 1,28 & 0,51 & 0,91 \\
\hline Coeficiente curtose & 1,12 & 6,67 & 0,85 & 9,57 & 16,01 & $-0,14$ \\
\hline Coeficiente assimetria & 1,2 & 2,27 & 0,95 & 2,74 & 2,69 & 1,04 \\
\hline
\end{tabular}

${ }^{(1)}$ Areia total. ${ }^{(2)} \mathrm{N}=$ número de dados.

Quadro 4. Parâmetros dos modelos de semivariogramas teóricos ajustados aos semivariogramas experimentais dos conjuntos amostrais por indicação para os atributos numéricos: teor de areia total (AT), teor de potássio trocável (K) e teor de alumínio trocável (Al)

\begin{tabular}{|c|c|c|c|c|c|c|c|c|}
\hline Corte & Valor & Direção & Modelo & Co & $C^{(1)}$ & Alcance & DE (2) & CDE (3) \\
\hline & & & & & & $\mathrm{km}$ & $\%$ & \\
\hline \multicolumn{9}{|c|}{$\mathrm{AT}, \mathrm{g} \mathrm{kg}^{-1}$} \\
\hline $1^{\circ}$ & 4,00 & Omnidirecional & Exponencial & 0,071 & 0,030 & 146,33 & 70 & $\mathrm{M}$ \\
\hline $2^{\circ}$ & 5,80 & Omnidirecional & Exponencial & 0,099 & 0,048 & 99,50 & 67 & $\mathrm{M}$ \\
\hline $3^{\circ}$ & 8,27 & Omnidirecional & Exponencial & 0,134 & 0,081 & 238,45 & 62 & M \\
\hline $4^{\circ}$ & 11,00 & Omnidirecional & Exponencial & 0,160 & 0,049 & 61,20 & 77 & W \\
\hline $5^{\circ}$ & 15,00 & Omnidirecional & Exponencial & 0,176 & 0,082 & 239,21 & 68 & M \\
\hline $6^{\circ}$ & 21,70 & Omnidirecional & Gaussiano & 0,198 & 0,051 & 248,32 & 80 & $\mathrm{~W}$ \\
\hline $7^{\circ}$ & 27,90 & Omnidirecional & Gaussiano & 0,175 & 0,037 & 231,16 & 83 & W \\
\hline $8^{\circ}$ & 37,60 & Omnidirecional & Exponencial & 0,100 & 0,048 & 36,86 & 68 & M \\
\hline $9^{\circ}$ & 46,70 & Omnidirecional & Exponencial & 0,076 & 0,015 & 18,37 & 84 & $\mathrm{~W}$ \\
\hline \multicolumn{9}{|c|}{$\mathrm{K}, \mathrm{mg} \mathrm{kg}^{-1}$} \\
\hline $1^{\circ}$ & 28 & Omnidirecional & Gaussiano & 0,074 & 0,017 & 30,22 & 81 & $\mathrm{~W}$ \\
\hline $2^{\circ}$ & 39 & Omnidirecional & Exponencial & 0,142 & 0,026 & 42,84 & 85 & $\mathrm{~W}$ \\
\hline $3^{\circ}$ & 49 & Omnidirecional & Exponencial & 0,165 & 0,047 & 143,14 & 78 & $\mathrm{~W}$ \\
\hline $4^{\circ}$ & 61 & Omnidirecional & Exponencial & 0,208 & 0,032 & 121,16 & 87 & W \\
\hline $5^{\circ}$ & 71 & Omnidirecional & Exponencial & 0,219 & 0,033 & 116,80 & 87 & W \\
\hline $6^{\circ}$ & 81 & Omnidirecional & Exponencial & 0,210 & 0,032 & 89,06 & 87 & $\mathrm{~W}$ \\
\hline $7^{\circ}$ & 100 & Omnidirecional & Exponencial & 0,183 & 0,022 & 57,59 & 89 & $\mathrm{~W}$ \\
\hline $8^{\circ}$ & 131 & Omnidirecional & Exponencial & 0,139 & 0,010 & 56,08 & 93 & W \\
\hline $9^{\circ}$ & 172 & Omnidirecional & Gaussiano & 0,070 & 0,003 & 30,95 & 96 & W \\
\hline \multicolumn{9}{|c|}{$\mathrm{Al}, \mathrm{cmol}_{\mathrm{c}} \mathrm{kg}^{-1}$} \\
\hline $1^{\circ}$ & 0,20 & Omnidirecional & Exponencial & 0,069 & 0,013 & 78,85 & 84 & $\mathrm{~W}$ \\
\hline $2^{\circ}$ & 0,60 & Omnidirecional & Exponencial & 0,095 & 0,028 & 53,51 & 77 & $\mathrm{~W}$ \\
\hline \multirow{2}{*}{$3^{\circ}$} & \multirow{2}{*}{1,30} & $100^{\circ}$ & Exponencial & 0,140 & 0,064 & 162,60 & 69 & M \\
\hline & & $10^{\circ}$ & Exponencial & 0,148 & 0,042 & 99,03 & 78 & W \\
\hline \multirow{2}{*}{$4^{\circ}$} & \multirow{2}{*}{2,19} & $100^{\circ}$ & Exponencial & 0,148 & 0,094 & 93,99 & 61 & $\mathrm{M}$ \\
\hline & & $10^{\circ}$ & Exponencial & 0,173 & 0,054 & 77,49 & 76 & W \\
\hline \multirow{2}{*}{$5^{\circ}$} & \multirow{2}{*}{2,90} & $100^{\circ}$ & Gaussiano & 0,182 & 0,073 & 52,55 & 71 & M \\
\hline & & $10^{\circ}$ & Gaussiano & 0,173 & 0,061 & 33,18 & 74 & M \\
\hline $6^{\circ}$ & 3,80 & Omnidirecional & Gaussiano & 0,172 & 0,084 & 60,08 & 67 & M \\
\hline $7^{\circ}$ & 4,60 & Omnidirecional & Gaussiano & 0,158 & 0,075 & 63,66 & 68 & $\mathrm{M}$ \\
\hline $8^{\circ}$ & 5,40 & Omnidirecional & Gaussiano & 0,133 & 0,056 & 61,51 & 70 & $\mathrm{M}$ \\
\hline $9^{\circ}$ & 7,10 & Omnidirecional & Es férico & 0,080 & 0,030 & 65,37 & 73 & M \\
\hline
\end{tabular}

${ }^{(1)} \mathrm{C}_{0}$ e $\mathrm{C}$ assumem a unidade do atributo elevada ao quadrado. ${ }^{(2)} \mathrm{DE}=100\left[\mathrm{Co} / \mathrm{C}_{0}+\mathrm{C}\right]^{(3)} \mathrm{Classe}$ de dependência espacial (cf. Cambardella et al., 1994), em que $\mathrm{W}=$ fraca dependência espacial ( $\mathrm{DE}>75 \%$ ), $\mathrm{M}=$ moderada dependência espacial $(75 \%>\mathrm{DE}>25 \%)$ e $\mathrm{S}=$ forte dependência espacial $(\mathrm{DE}<25 \%)$. 
Quadro 5. Parâmetros dos modelos de semivariogramas teóricos ajustados aos semivariogramas experimentais dos conjuntos amostrais por indicação para os atributos numéricos: soma de bases (S), capacidade de troca catiônica (CTC) e saturação por bases (V)

\begin{tabular}{|c|c|c|c|c|c|c|c|c|}
\hline Corte & Valor & Direção & Modelo & Co & $C^{(1)}$ & Alcance & DE (2) & CDE (3) \\
\hline & & & & & & $\mathrm{m}$ & $\%$ & \\
\hline \multicolumn{9}{|c|}{$\mathrm{S}, \mathrm{cmol}_{\mathrm{c}} \mathrm{kg}^{-1}$} \\
\hline $1^{\mathrm{o}}(4)$ & 0,70 & Omnidirecional & Exponencial & 12,355 & 3,051 & 42,88 & 80 & W \\
\hline $2^{\circ}$ & 0,98 & Omnidirecional & Gaussiano & 0,153 & 0,020 & 39,36 & 88 & W \\
\hline $3^{\circ}$ & 1,32 & Omnidirecional & Esférico & 0,180 & 0,044 & 42,30 & 80 & W \\
\hline $4^{\circ}$ & 1,77 & Omnidirecional & Esférico & 0,219 & 0,027 & 58,99 & 89 & W \\
\hline \multirow{2}{*}{$5^{\circ}$} & \multirow{2}{*}{2,14} & $100^{\circ}$ & Esférico & 0,212 & 0,034 & 33,41 & 86 & W \\
\hline & & $10^{\circ}$ & Es férico & 0,156 & 0,099 & 16,12 & 61 & M \\
\hline $6^{\circ}$ & 2,85 & Omnidirecional & Exponencial & 0,195 & 0,039 & 79,02 & 83 & W \\
\hline $7^{\circ}$ & 4,20 & Omnidirecional & Exponencial & 0,142 & 0,050 & 48,83 & 74 & M \\
\hline $8^{\circ}$ & 6,41 & Omnidirecional & Exponencial & 0,108 & 0,013 & 61,92 & 89 & W \\
\hline $9^{\circ}$ & 11,80 & Omnidirecional & Gaussiano & 0,058 & 0,010 & 40,15 & 85 & W \\
\hline \multicolumn{9}{|c|}{$\mathrm{CTC}, \mathrm{cmol}_{\mathrm{c}} \mathrm{kg}^{-1}$} \\
\hline $1^{\circ}$ & 7,00 & Omnidirecional & Exponencial & 0,035 & 0,061 & 149,69 & 36 & M \\
\hline $2^{\circ}$ & 10,49 & Omnidirecional & Exponencial & 0,070 & 0,089 & 131,66 & 44 & M \\
\hline $3^{\circ}$ & 12,25 & Omnidirecional & Exponencial & 0,128 & 0,093 & 242,28 & 58 & M \\
\hline \multirow{2}{*}{$4^{\circ}$} & \multirow{2}{*}{14,19} & $107^{\circ}$ & Exponencial & 0,171 & 0,064 & 121,30 & 73 & M \\
\hline & & $17^{\circ}$ & Exponencial & 0,132 & 0,102 & 64,77 & 56 & M \\
\hline \multirow{2}{*}{$5^{\circ}$} & \multirow{2}{*}{15,54} & $107^{\circ}$ & Exponencial & 0,162 & 0,094 & 89,31 & 63 & M \\
\hline & & $17^{\circ}$ & Exponencial & 0,105 & 0,137 & 74,46 & 43 & M \\
\hline $6^{\circ}$ & 17,08 & Omnidirecional & Exponencial & 0,140 & 0,096 & 47,79 & 59 & M \\
\hline $7^{\circ}$ & 18,91 & Omnidirecional & Exponencial & 0,141 & 0,069 & 79,23 & 67 & M \\
\hline $8^{\circ}$ & 21,06 & Omnidirecional & Exponencial & 0,120 & 0,035 & 46,80 & 77 & W \\
\hline $9^{\circ}$ & 24,67 & Omnidirecional & Gaussiano & 0,070 & 0,017 & 82,81 & 80 & $\mathrm{~W}$ \\
\hline \multicolumn{9}{|c|}{$\mathrm{V}, \%$} \\
\hline $1^{\mathrm{o}}(4)$ & 4,26 & Omnidirecional & Esférico & 12,052 & 4,400 & 84,30 & 73 & M \\
\hline $2^{\circ}$ & 6,81 & Omnidirecional & Gaussiano & 0,144 & 0,037 & 80,64 & 80 & $\mathrm{~W}$ \\
\hline \multirow{2}{*}{$3^{\circ}$} & \multirow{2}{*}{8,89} & $115^{\circ}$ & Exponencial & 0,161 & 0,084 & 134,78 & 66 & M \\
\hline & & $25^{\circ}$ & Exponencial & 0,149 & 0,068 & 76,65 & 69 & M \\
\hline \multirow{2}{*}{$4^{\circ}$} & \multirow{2}{*}{12,09} & $115^{\circ}$ & Esférico & 0,195 & 0,068 & 112,44 & 74 & M \\
\hline & & $25^{\circ}$ & Es férico & 0,173 & 0,063 & 60,32 & 73 & M \\
\hline \multirow{2}{*}{$5^{\circ}$} & \multirow{2}{*}{16,86} & $115^{\circ}$ & Exponencial & 0,183 & 0,090 & 174,88 & 67 & M \\
\hline & & $25^{\circ}$ & Exponencial & 0,165 & 0,081 & 100,11 & 67 & M \\
\hline \multirow{2}{*}{$6^{\circ}$} & \multirow{2}{*}{22,30} & $108^{\circ}$ & Exponencial & 0,151 & 0,092 & 106,36 & 62 & M \\
\hline & & $18^{\circ}$ & Exponencial & 0,156 & 0,070 & 60,98 & 69 & M \\
\hline $7^{\circ}$ & 32,86 & Omnidirecional & Exponencial & 0,147 & 0,056 & 134,74 & 72 & M \\
\hline $8^{\circ}$ & 49,83 & Omnidirecional & Exponencial & 0,120 & 0,026 & 161,71 & 82 & $\mathrm{~W}$ \\
\hline $9^{\circ}$ & 69,49 & Omnidirecional & Esférico & 0,070 & 0,007 & 325,60 & 91 & $\mathrm{~W}$ \\
\hline
\end{tabular}

${ }^{(1)} \mathrm{C}_{\mathrm{o}}$ e $\mathrm{C}$ assumem a unidade do atributo elevada ao quadrado. ${ }^{(2)} \mathrm{DE}=100\left[\mathrm{Co} / \mathrm{C}_{\mathrm{o}}+\mathrm{C}\right]$. $^{(3)} \mathrm{Classe}$ de dependência espacial (cf. Cambardella et al., 1994), em que $\mathrm{W}=$ fraca dependência espacial ( $\mathrm{DE}>75 \%), \mathrm{M}=$ moderada dependência espacial (75\% > DE > 25\%) e $\mathrm{S}=$ forte dependência espacial $(\mathrm{DE}<25 \%) \cdot{ }^{4} \mathrm{O}$ variograma experimental estimado para o conjunto amostral foi o semivariograma relativo geral.

variabilidade espacial. $\mathrm{Na}$ inferência espacial de atributos categóricos (Bönisch, 2004), Al e V também apresentaram anisotropia nos semivariogramas ajustados em algumas classes. O caráter anisotrópico do semivariograma indicou que a variabilidade espacial variou de acordo com a direção, e este aspecto pode ser útil na definição de formas de amostragem desses atributos.

Outro aspecto que se destacou nos semivariogramas ajustados (Quadros 4 e 5) foram os valores de efeito pepita. Quando comparados entre si, o efeito pepita foi maior que a variância estrutural, em todos os semivariogramas ajustados, com exceção de alguns obtidos para CTC (Quadros 4 e 5). Oatributo $\mathrm{K}$ apresentou efeito pepita 14 e 23 vezes maior que a contribuição no $8^{\circ}$ e $9^{\circ}$ cortes, respectivamente. Como o efeito pepita é o valor da semivariância para a distância zero (Vieira, 2000) e representa o componente da variabilidade espacial que não pode ser relacionado com uma causa específica (variabilidade ao acaso), constatou-se que o número de dados utilizados neste estudo não foi suficiente para descrever a variabilidade espacial dos atributos considerados. Neste caso, uma densidade maior de dados certamente seria mais adequada, mas não necessariamente mais accessível. Por outro lado, o 
uso de informações secundárias ou de procedimentos de cokrigagem, krigagem regressiva, krigagem fatorial, etc., bem como de outros métodos inferenciais (baysianos, funções de pedotranferência, dentre outros), poderiam aumentar a acurácia das estimativas (Goovaerts, 2000; McBratney et al., 2000).

Comparando o atributo $\mathrm{K}$, cujo conteúdo no solo pode ser modificado por práticas de adubação, com a CTC, cujos valores são dependentes da quantidade e tipo de colóides minerais e orgânicos no solo, constatou-se que no primeiro a variabilidade ao acaso é muito maior do que a variabilidade espacial, enquanto, na CTC, constatou-se uma mediana dependência espacial, no conjunto de dados aqui tratados. Cabe destacar que as diferentes origens dos dados utilizados podem interferir fortemente nos resultados obtidos, visto que uma premissa para computar um semivariograma é assumir que todos os dados estão localizados numa mesma classe ou domínio, conforme destacaram Burrough \& McDonnel (1998). De todo modo, este aspecto chamou a atenção para o forte componente ao acaso da variabilidade espacial de atributos que podem ser modificados pelo uso do solo.

Couto et al. (2000), utilizando krigagem ordinária na interpolação de valores de $\mathrm{K}$ em solos do Mato Grosso, já haviam constatado que o efeito pepita nos horizontes A era maior do que nos horizontes B, pois as práticas de manejo, que influem principalmente no horizonte A, condicionaram diferentes comportamentos na estrutura espacial do atributo.

No presente trabalho, os dados foram tratados, levando-se em conta a forma como as raízes exploram os horizontes do solo numa cultura da soja, adotando-se pesos para os valores dos atributos em cada horizonte ou camada de acordo com critérios de distribuição de raízes propostos por Miyasaka \& Medina (1981). Assim, a variografia de cada atributo correspondeu ao seu comportamento espacial do solo como um todo em relação à cultura de soja e, da observação dos quadros 4 e 5, pôde-se constatar que as variabilidades observadas refletiram, de modo geral, o comportamento do atributo representado.

No conjunto de dados estudados no estado de Santa Catarina, os valores de alcance, que representam a distância da origem a partir da qual não existe mais dependência espacial entre as amostras, variaram tanto de um atributo para outro, quanto nos diferentes intervalos de corte de cada atributo. Webster (1985) destaca que os alcances obtidos nos semivariogramas têm uma importância muito grande na determinação do limite de dependência espacial. Os resultados obtidos evidenciaram que os limites de dependência espacial dos atributos $\mathrm{K}$, $\mathrm{Al}, \mathrm{S}, \mathrm{V}, \mathrm{CTC}$ e AT variaram de acordo com a estrutura do atributo (variação do alcance entre atributos) e com o tipo de solo (variação do alcance de acordo com o intervalo de corte).
As espacializações dos atributos e de suas incertezas associadas, expressadas em intervalos de confiança de $95 \%$ de probabilidade, estão apresentadas nas figuras 3 e 4 . Tomando como exemplo as figuras $3 \mathrm{a}$ e $3 \mathrm{~b}$, tem-se em $3 \mathrm{a}$ a espacialização do atributo AT e em $3 \mathrm{~b}$ a incerteza associada. Constata-se que os teores de areia total (AT) no estado de Santa Catarina, inferidos a partir dos dados de AT utilizados para a predição espacial, variaram de $4 \%$ a $51 \%$ (Quadro 3) e esses valores, representados em níveis de cinza, são tanto maiores quanto mais clara for a área. Por outro lado, a incerteza associada, também representada em níveis de cinza, variou de 4 a $58 \%$. Ou seja, existe uma probabilidade de $95 \%$ do valor estimado para o teor de areia total em pontos não amostrados do estado de Santa Catarina estar situado no intervalo [4-51 \%], com desvio-padrão [2-29\%]. Portanto, nas figuras 3 e 4 , as representações na coluna da esquerda indicam que, quanto mais clara for a área, maior o valor do atributo considerado, enquanto, nas representações da direita, quanto mais clara for a área, maior a incerteza (expressa em termos de $2 \sigma$ ) do valor estimado. Calculando o desvio-padrão em relação à média (Quadro 6), verificou-se que para o mesmo valor médio estimado (mesmo tom de cinza nas representações a, c e e das figuras 3 e 4), o CV é tanto maior quanto mais clara for a área na representação das incertezas associadas. Constatase também que os CVs dos valores estimados podem ser muito altos (áreas escuras nas representações a, c e e com áreas claras nas representações b, d e f), alcançando até $1096 \%$ na soma de bases (Quadro 6), muito superior ao indicado por Vauclin (1983).

No caso do estado de Santa Catarina, e considerando os dados e os procedimentos inferenciais utilizados, a propagação de incertezas variou de acordo com o tipo de atributo estudado e da área considerada, confirmando que a variabilidade espacial depende do comportamento do atributo e da combinação de fatores ambientais. Por exemplo, no caso de AT (Figura 3a), verifica-se que, na parte oeste do estado (à esquerda da linha pontilhada), predominam solos com teores de areia estimados entre 4 e $27 \%$ (zonas escuras a cinza), e a incerteza associada, expressa em valores de $2 \sigma$, situa-se entre 4 e $30,9 \%$ (zonas escuras a cinzas), conferindo CV entre 7,6 e 369,8 \% (Quadro 6). Por outro lado, na porção leste do estado (à direita da linha pontilhada), solos com teores de areia estimados entre 27 e $51 \%$ (zonas cinzas a claras) foram predominantes e a incerteza associada situou-se entre 30,9 e $57 \%$ (zonas cinzas a claras), predominando, portanto, $\mathrm{CV}$ entre 30 e $104 \%$ (Quadro 6).

Adotando o mesmo procedimento de análise detalhada do quadro 6 e dos demais atributos representados nas figuras 3 e 4 , verificou-se que AT e CTC apresentaram maior predominância de áreas de CV médio a baixo na parte leste do estado de Santa Catarina do que na parte oeste. Por outro 
(a)

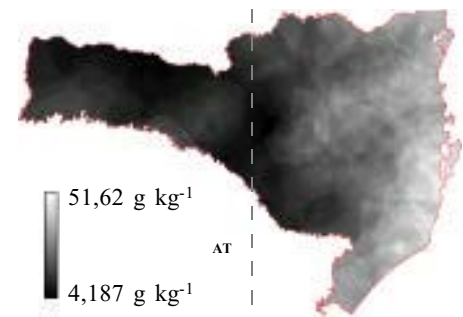

(c)

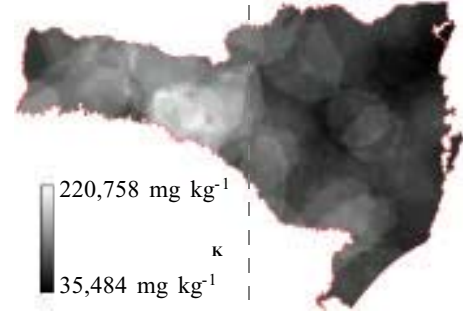

(e)

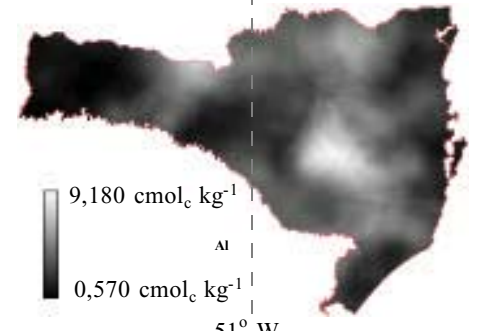

(b)

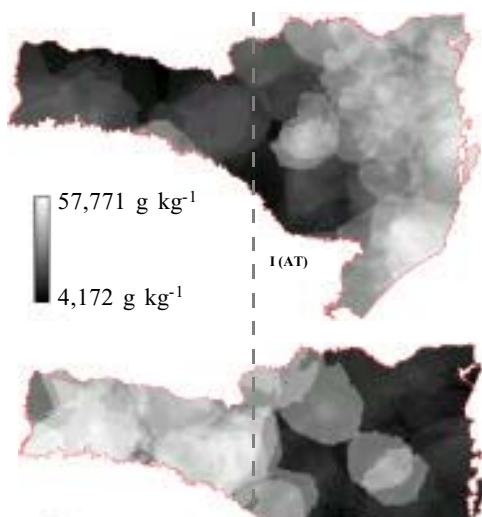

(f)

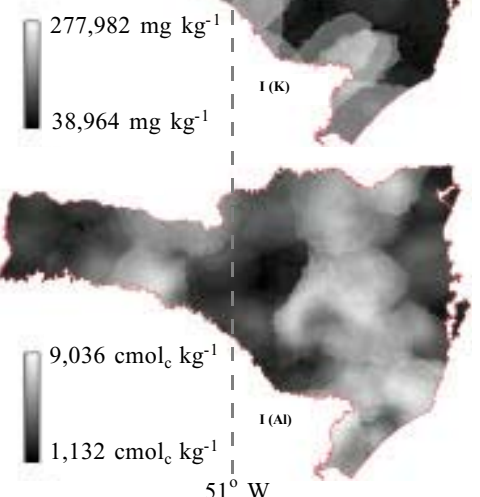

Figura 3. Espacialização de atributos numéricos e incertezas associadas: teor de areia total [(AT) e I(AT)], teor de potássio trocável $[(\mathrm{K})$ e I(K)] e teor de alumínio trocável [(Al) e I(Al)]. A linha pontilhada indica o meridiano $51^{\circ} \mathrm{W}$ que divide o estado de Santa Catarina aproximadamente ao meio.

(a)

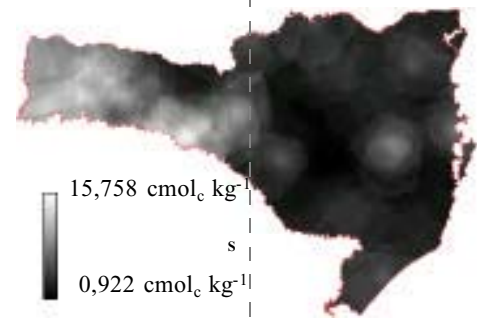

(c)

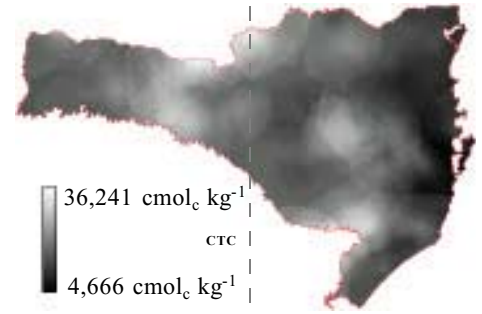

(e)

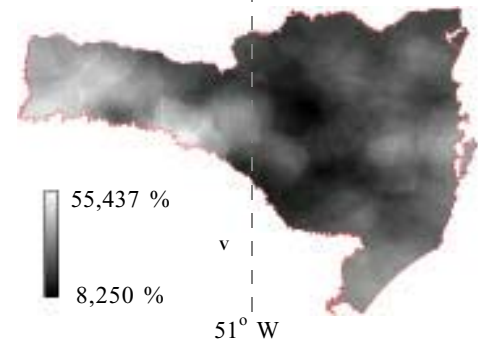

(b)

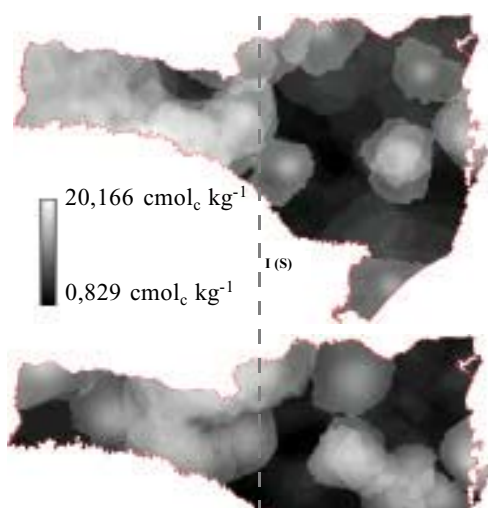

(d)

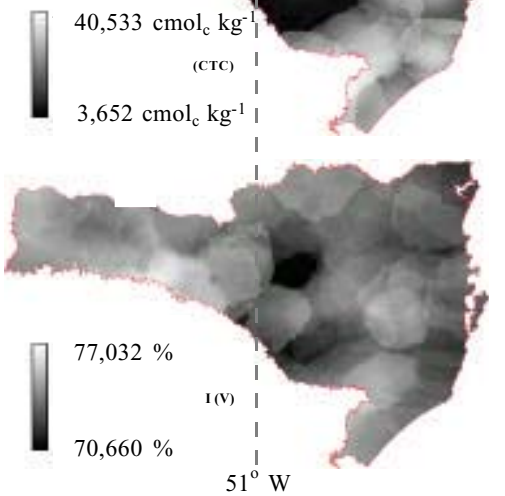

Figura 4. Espacialização de atributos numéricos e incertezas associadas: soma de bases $[(\mathbf{S})$ e $\mathbf{I}(\mathbf{S})]$, capacidade de troca catiônica $[(C T C)$ e I(CTC)] saturação por bases $[(V)$ e I(V)]. A linha pontilhada indica o meridiano $51^{\circ} \mathrm{W}$ que divide o estado de Santa Catarina aproximadamente ao meio. 
Quadro 6. Coeficientes de variação de atributos numéricos ${ }^{(1)}$ de solos e suas incertezas associadas, tomando por referência as diferentes

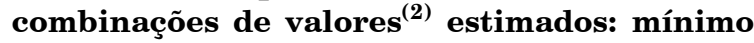
(área escura), médio (área cinza médio) e máximo (área clara) e incertezas ${ }^{(3)}$ mínima (área escura), média (área cinza médio) e máxima (área clara) das representações obtidas

\begin{tabular}{|c|c|c|c|}
\hline \multirow[b]{2}{*}{ Inc. $(2 \sigma)$} & \multicolumn{3}{|c|}{$C V^{(4)}(\%)$} \\
\hline & Escura & $\begin{array}{l}\text { Cinza } \\
\text { médio }\end{array}$ & Clara \\
\hline & \multicolumn{3}{|c|}{$\mathrm{AT}, \mathrm{g} \mathrm{kg}^{-1}$} \\
\hline & $(4,187)$ & $(27,624)$ & $(51,062)$ \\
\hline Escura $(4,172)$ & 49,8 & 7,6 & 4,1 \\
\hline Cinza médio $(30,972)$ & 369,8 & 56 & 30,3 \\
\hline \multirow[t]{3}{*}{ Clara $(57,771)$} & 689,9 & 104,6 & 56,6 \\
\hline & \multicolumn{3}{|c|}{$\mathrm{K}, \mathrm{mg} \mathrm{kg}^{-1}$} \\
\hline & $(35,484)$ & $(128,121)$ & $(220,758)$ \\
\hline Escura $(38,964)$ & 54,9 & 15,2 & 8,8 \\
\hline Cinza médio $(158,473)$ & 223,3 & 61,8 & 35,9 \\
\hline \multirow[t]{3}{*}{ Clara $(277,982)$} & 391,7 & 108,5 & 63 \\
\hline & \multicolumn{3}{|c|}{$\mathrm{Al}, \mathrm{cmol}_{\mathrm{c}} \mathrm{kg}^{-1}$} \\
\hline & $(0,570)$ & $(4,875)$ & $(9,180)$ \\
\hline Escura $(1,132)$ & 99,3 & 11,6 & 6,2 \\
\hline Cinza médio $(5,084)$ & 446 & 52,1 & 27,7 \\
\hline \multirow[t]{3}{*}{ Clara $(9,036)$} & 792,6 & 92,7 & 49,2 \\
\hline & \multicolumn{3}{|c|}{$\mathrm{S}, \mathrm{cmol}_{\mathrm{c}} \mathrm{kg}^{-1}$} \\
\hline & $(0,922)$ & $(8,34)$ & $(15,758)$ \\
\hline Escura $(0,829)$ & 22,4 & 5 & 2,6 \\
\hline Cinza médio $(10,498)$ & 569,4 & 62,9 & 33,3 \\
\hline \multirow[t]{3}{*}{ Clara $(20,166)$} & 1093,6 & 120,9 & 64 \\
\hline & \multicolumn{3}{|c|}{$\mathrm{CTC}, \mathrm{cmol}_{\mathrm{c}} \mathrm{kg}^{-1}$} \\
\hline & $(4,666)$ & $(20,454)$ & $(36,241)$ \\
\hline Escura $(3,652)$ & 39,1 & 8,9 & 5 \\
\hline Cinza médio $(22,092)$ & 237 & 54 & 30,5 \\
\hline \multirow[t]{3}{*}{ Clara $(40,533)$} & 443 & 101 & 57 \\
\hline & \multicolumn{3}{|c|}{$\mathrm{V}, \%$} \\
\hline & $(8,25)$ & $(31,844)$ & $(55,437)$ \\
\hline Escura $(10,66)$ & 64,6 & 16,7 & 9,6 \\
\hline Cinza médio $(43,846)$ & 265,7 & 68,8 & 39,5 \\
\hline Clara $(77,032)$ & 466,9 & 121 & 69,5 \\
\hline
\end{tabular}

(1) Teor de areia total (AT), teor de potássio (K), teor de alumínio trocável (Al), soma de bases (S), capacidade de troca catiônica (CTC), saturação por bases (V). ${ }^{(2)}$ Valores estimados $(\mu)$ entre parênteses nas linhas. ${ }^{(3)}$ Incertezas estimadas $(2 \sigma)$ com grau de confiança de $95 \%$ de probabilidade e indicadas entre parênteses na primeira coluna. ${ }^{(4)} \mathrm{CV}=100$ [Incerteza/(2 x Valor $\left.)\right]$.

lado, com os atributos S, K e V houve maior predominância de áreas de CV médio a baixo na parte oeste do estado de Santa Catarina do que na parte leste. Considerando que os perfis e as amostras de solos utilizados neste trabalho concentravam-se principalmente na parte leste do estado e que esses dados foram obtidos em períodos distintos, supõe-se que, quando as variáveis de solos são modificáveis pelo uso, como é o caso de $\mathrm{K}$, S e V, um maior número de dados levou a uma maior incerteza na inferência espacial, enquanto, em atributos não afetados pelo uso, o maior número de dados diminuiu o grau de incerteza. $\mathrm{O} \mathrm{Al}$ teve um comportamento intermediário, pois, na espacialização de dados estimados e de suas incertezas associadas, os CVs foram amplos tanto a leste quanto a oeste de Santa Catarina.

$\mathrm{Na}$ espacialização da limitação das terras em fertilidade, os atributos $\mathrm{K}, \mathrm{Al}, \mathrm{S}, \mathrm{CTC}$ e V foram expressos, inicialmente, de acordo com a aptidão em fertilidade, adotando-se valores indicados no quadro 1 para os parâmetros da função linear para classificação contínua no mapeamento fuzzy (Quadro 2). As figuras 5 e 6 mostram os resultados das classificações contínuas das propriedades químicas e as incertezas propagadas, expressas em intervalos de confiança de $95 \%$. Esses resultados indicaram que a incerteza dos atributos K e CTC diminuiu quando essas representações foram expressas de acordo com a fertilidade. Por outro lado, as incertezas dos atributos $\mathrm{Al}, \mathrm{S}$ e $\mathrm{V}$ expressos em função da fertilidade foram diferenciadas. Constatou-se que de modo geral, a incerteza da estimativa de fertilidade diminuiu em relação às representações parciais dos atributos estudados expressos em termos de fertilidade (Figura 6c). Isto ocorreu provavelmente porque, ao se dar o mesmo peso $(0,2)$ a todos os atributos, suavizaram-se os efeitos de cada um dos atributos na estimativa de fertilidade.

No estado de Santa Catarina, predominam solos de baixa fertilidade, ou seja, com $\mathrm{S}$ e $\mathrm{V}$ baixos e $\mathrm{Al}$ altos. Isto fez com que predominassem valores baixos de $\mathrm{S}$ e de $\mathrm{V}$ e valores altos de $\mathrm{Al}$ na distribuição de freqüência de valores, o que refletiu na incerteza dos dados quando o valor inferido apontava para teores altos de $\mathrm{S}$ e de $\mathrm{V}$ e teores muito baixos de $\mathrm{Al}$.

A diminuição da incerteza na representação de K e CTC em fertilidade chamou atenção para dois aspectos interessantes. Os perfis e as amostras estudados referiam-se a solos com valores de CTC e de $\mathrm{K}$ suficientes para permitir representações nas duas classes de fertilidade previstas para essas variáveis. Como em solos de baixa fertilidade a CTC é predominantemente baixa, pode-se afirmar que a variabilidade espacial da fertilidade do solo é mais bem representada em termos de CTC do que pelos atributos $\mathrm{S}, \mathrm{Al}$ e V, que, conforme já destacado, podem ser modificados pelo uso. Ou seja, como a inferência de um atributo para uma localização $u$ não conhecida depende do valor do atributo na localização u conhecida (medida), atributos que podem ser modificados no tempo pela ação do homem aumentam o grau de incerteza dos dados, visto que podem ter valores muito distintos mesmo quando situados relativamente próximos, principalmente se os dados foram coletados em épocas diferentes, como é o caso do presente estudo. 

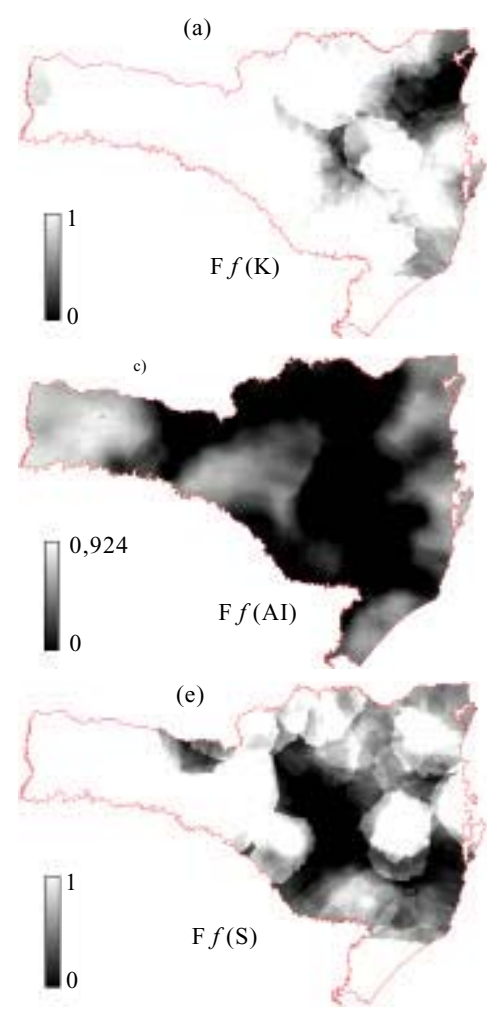
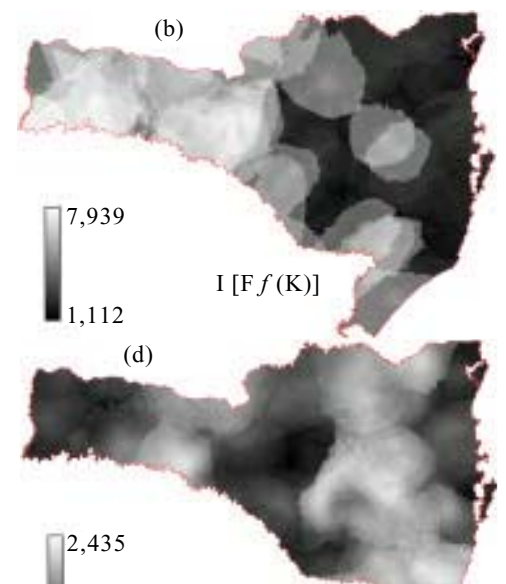

$\mathrm{I}[\mathrm{F} f(\mathrm{AI})]$

0,305

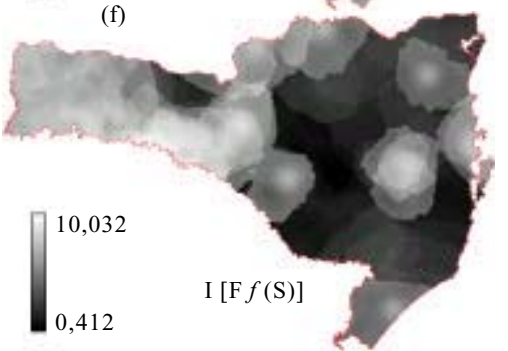

Figura 5. Espacialização de fertilidade numérica e incertezas associadas aos atributos: teor de potássio trocável $[\mathrm{F} f(\mathrm{~K})$ e I $[\mathrm{F} f(\mathrm{~K})]]$, teor de alumínio trocável $[\mathrm{F} f(\mathrm{Al})$ e $\mathrm{I}[\mathrm{F} f(\mathrm{Al})]]$ e soma de bases $[\mathrm{F} f(\mathrm{~S})$ e $\mathrm{I}[\mathrm{F} f(\mathrm{~S})]]$, expressas em valores adimensionais.
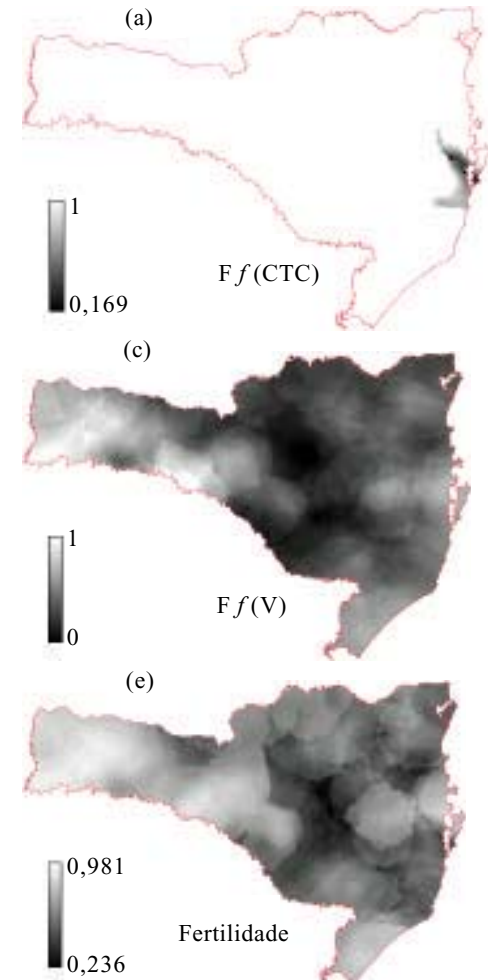
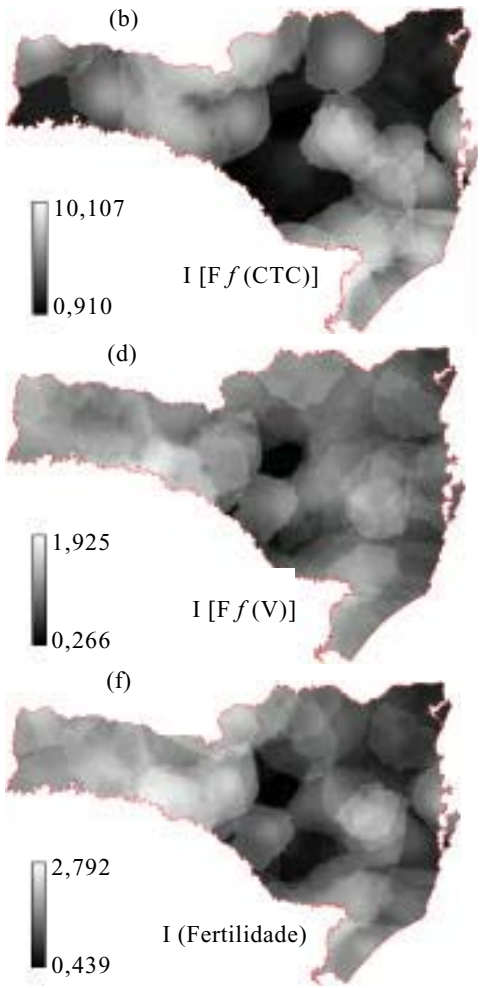

Figura 6. Espacialização de fertilidade numérica e incertezas associadas aos atributos: capacidade de troca catiônica $[F f(C T C)$ e $I[F f(C T C)]]$ e saturação por bases $[F f(V)$ e $I[F f(V)]]$ e espacialização associada à álgebra de mapas gerando a representação da limitação em fertilidade e incerteza associada [Fertilidade e I(Fertilidade)], expressas em valores adimensionais. 
$\mathrm{O}$ segundo aspecto a ser destacado diz respeito ao teor de K. Embora este seja utilizado no cálculo de S, a incerteza da fertilidade expressa em função de $\mathrm{K}$ foi menor do que a incerteza da fertilidade expressa em função de S. Conforme já destacado, o $\mathrm{K}$ apresentou variabilidade ao acaso maior do que a variabilidade espacial. Isto se deve provavelmente às mudanças ocorridas ao longo do tempo em função do uso do solo (amostras coletadas em épocas diferentes). Conforme Duchaufour (1991): (a) a participação de $\mathrm{K}$ no valor de $\mathrm{S}\left(=\mathrm{Ca}^{2+}+\mathrm{Mg}^{2+}+\mathrm{K}^{+}+\right.$ $\mathrm{Na}^{+}+\mathrm{NH}_{4}^{+}$) é menor do que a dos íons $\mathrm{Ca}^{2+} \mathrm{e} \mathrm{Mg}^{2+}$; (b) $\mathrm{Ca}^{2+} \mathrm{e} \mathrm{Mg}^{2+}$ apresentam menor poder de troca do que o $\mathrm{K}^{+}$; (c) o íon $\mathrm{K}^{+}$é mais solúvel do que os íons $\mathrm{Ca}^{2+} \mathrm{e} \mathrm{Mg}^{2+}$; (d) as necessidades das plantas em $\mathrm{K}^{+}$ são, em geral, maiores do que as de $\mathrm{Mg}^{2+} \mathrm{e}$ menores do que as de $\mathrm{Ca}^{2+} ; \mathrm{v}$ ) em geral, $\mathrm{Na}^{+}$aparece nos solos em teores ínfimos (exceto em solos salinos) e não é indispensável às plantas (exceto para halófitas e algumas espécies de plantas $\mathrm{C}_{4}$ ), enquanto $\mathrm{NH}_{4}{ }^{+}$tem duração efêmera no solo.

Portanto, acredita-se que a expressão do teor de $\mathrm{K}^{+}$solúvel (atributo K neste trabalho) em termos de classe de fertilidade envolva menor imprecisão do que a da soma de bases (S) porque, se o solo tiver sido adubado com K, o efeito dessa adubação será menos duradouro do que da adubação com bases bivalentes; com isso, dados de perfis e amostras de solos situados próximos no espaço, coletados antes da adubação e dados coletados depois da adubação (dados de épocas distintas), diferirão menos se avaliados pelo conteúdo de K. Embora os estudos estatísticos não possam ser tomados como prova de relações de causa-efeito, os resultados obtidos com as incertezas das representações de $\mathrm{K}$ e $\mathrm{S}$ expressos em termos de classe de fertilidade parecem corroborar esta hipótese.

\section{CONCLUSÕES}

1. A krigagem por indicação permitiu espacializar, como geocampos numéricos, os atributos de solos teor de potássio (K), teor de alumínio trocável (Al), saturação por bases (V), soma de bases (S), capacidade de troca catiônica (CTC) e areia total (AT); gerar, para cada representação numérica, uma medida espacial de incerteza, e modelar a propagação de incerteza gerada em procedimentos booleanos de álgebra de mapas de atributos numéricos.

2. Os limites de dependência espacial dos atributos: $\mathrm{K}, \mathrm{Al}, \mathrm{S}, \mathrm{V}, \mathrm{CTC}$ e AT variaram de acordo com a estrutura do atributo (variação do alcance entre atributos) e da classe de solo (variação do alcance em função do intervalo de corte).

3. AT e CTC apresentaram maior predominância de áreas de CV médio a baixo na parte leste do estado de Santa Catarina do que na parte oeste, enquanto com os atributos $\mathrm{S}, \mathrm{KeV}$ houve maior predominância de áreas de CV médio a baixo na parte oeste do estado de Santa Catarina do que na parte leste.

4. Em atributos de solos modificáveis pelo uso, como é o caso de $\mathrm{K}, \mathrm{S}$ e $\mathrm{V}$, um maior número de dados numéricos levou a uma maior confusão na inferência espacial (maior incerteza), enquanto em atributos não afetados pelo uso o maior número de dados numéricos diminuiu o grau de incerteza.

5. Os atributos $\mathrm{Al}, \mathrm{S} \mathrm{e} \mathrm{V}$, quando expressos em classe de fertilidade, apresentaram aumento da incerteza quando os teores indicavam solos de fertilidade alta (teores de $\mathrm{Al}$ muito baixos, valores de $\mathrm{S}$ médios e altos, $\mathrm{V}$ alto).

6. Os atributos K e CTC, quando expressos de acordo com a classe de fertilidade, apresentaram diminuição da incerteza, expressa em intervalos de confiança de 95\%, e, conseqüentemente, diminuição do $\mathrm{CV}$ em relação à média.

7. Houve diminuição da incerteza quando diferentes representações numéricas foram integradas por procedimentos fuzzy de álgebra de mapas provavelmente em virtude da suavização dos efeitos de cada um dos atributos na estimativa de fertilidade causada pela adoção de um mesmo peso para todos os atributos.

8. Recomenda-se que, na representação e integração de atributos numéricos, visando à avaliação da fertilidade do solo para fins de planejamento regional, sejam observados aspectos como o comportamento espacial do atributo, a possibilidade de modificação de valores de acordo com o uso e o tipo de representação espacial (categórica ou numérica), a fim de obter modelos com menor grau de incerteza.

\section{LITERATURA CITADA}

ALMEIDA, J.A. Projeto pastagens nativas. Lages, Empresa Catarinense de Pesquisa Agropecuária e Universidade do estado de Santa Catarina, 1988. Não publicado.

ALMEIDA, J.A.; KÄMPF, N. \& ALMEIDA, R. Caracterização mineralógica de Cambissolos originados de rochas pelíticas nos patamares do Alto Rio Itajaí e no Planalto de Lages (SC). R. Bras. Ci. Solo, 21:181-190, 1997.

BARBOSA, C.C.F. Álgebra de mapas e suas aplicações em sensoriamento remoto e geoprocessamento. São José dos Campos, Instituto Nacional de Pesquisas Espaciais, 1997. 110p. (Tese de Mestrado)

BERRY, J.K. Cartographic modeling: the analytical capabilities of GIS. In: GOODCHILD, M.; PARKS, B.O. \& STEYAERT, L.T., eds. Environmental modeling with GIS. Oxford, Oxford University Press, 1993. p.58-74.

BOGNOLA, I.A. Caracterização química, física e mineralógica de solos intermediários entre Latossolos Brunos e Latossolos Roxos. Viçosa, Universidade Federal de Viçosa, 1995. 215p. (Tese de Mestrado) 
BONHAM-CARTER, G.F. Geographical information systems for geoscientists: modelling with GIS. New York, 1996. 398p. (Computer Methods in Geosciences, 13)

BÖNISCH, S.; LOPES ASSAD, M.L.; CÂMARA, G. \& MONTEIRO, A.M.V. Representação e propagação de incertezas em dados de solo. I - atributos categóricos. R. Bras. Ci. Solo, 27:21-32, 2004.

BURROUGH, P.A. \& McDONNEL, R.A. Principles of geographic information systems. New York, Oxford University Press, 1998. 333p.

CAMBARDELLA, C.A.; MOORMAN, T.B.; NOVACK, J.M.; PARKIN, T.B.; KARLEN, D.L.; TURCO, R.F. \& KNOPKA, A.E. Field-scale variability of soil properties in central Iowa soils. Soil Sci. Am. J., 58:1240-1248, 1994.

CORRÊA, J. Mineralogia e gênese das principais classes de solos de encostas basálticas no estado de Santa Catarina. Lages, Universidade do Estado de Santa Catarina, 2001. 165p. (Tese de Mestrado)

COUTO, E.G.; KLAMT, E. \& STEIN, A. Estimativa do teor de argila e do potássio trocável em solos esparsamente amostrados no sul do Estado do Mato Grosso. R. Bras. Ci. Solo, 24:129-140, 2000.

DEUTSCH, C.V. \& JOURNEL, A.G. GSLIB: Geostatistical software library and user's guide. New York, Oxford University Press, 1998. 369p.

DUCHAUFOUR, Ph. Pédologie: sol, végétation, environnement. Paris, Masson, 1991. 289 p.

EMPRESA BRASILEIRA DE PESQUISA AGROPECUÁRIA EMBRAPA. Centro Nacional de Pesquisas de Solos. Levantamento de reconhecimento de alta intensidade dos solos do estado de Santa Catarina. Rio de Janeiro, EMBRAPA, 1998. Não publicado.

EMPRESA BRASILEIRA DE PESQUISA AGROPECUÁRIA EMBRAPA. Centro Nacional de Pesquisas de Solos. Zoneamento pedoclimático do estado de Santa Catarina. Rio de Janeiro, 1997. 59p.

FELGUEIRAS, C.A. Modelagem ambiental com tratamento de incertezas em sistemas de informações geográficas: paradigma geoestatístico por indicação. São José dos Campos, Instituto Nacional de Pesquisas Espaciais, 1999. 181 p. (Tese de Doutorado)

FUKS, S.D. Novos modelos para mapas derivados de informações de solos. In: ASSAD, E.D. \& SANO, E.E., eds. Sistemas de informações geográficas: aplicações na agricultura. Brasília, Empresa Brasileira de Pesquisa Agropecuária - SPI, 1998. $434 p$.
GMACH, J. Evolução mineralógica dos solos e relações solosuperfícies geomórficas em áreas de encostas basálticas no Vale do Rio do Peixe, SC: toposseqüência de Ipira - Peritiba. Lages, Universidade do Estado de Santa Catarina, 1999. 142p. (Tese de Mestrado)

GOOVAERTS, P. Geostatistical modelling of uncertainty in soil science. Geoderma, 103:3-26, 2001.

HEUVELINK, G.B.M. Error propagation in environmental modelling with GIS. London, Taylor \& Francis, 1998. 127p.

INSTITUTO BRASILEIRO DE GEOGRAFIA E ESTATÍSTICA - IBGE. Levantamento de recursos naturais. Rio de Janeiro, 1986, 796p.

INSTITUTO BRASILEIRO DE GEOGRAFIA E ESTATÍSTICA - IBGE. Levantamento de recursos naturais. Rio de Janeiro, 1999, v.35. Não publicado.

ISAAKS, E.H. \& SRIVASTAVA, R.M. An introduction to applied geostatistics. New York, Oxford University Press, 1989. $561 \mathrm{p}$.

McBRATNEY, A.B.; ODEH, I.O.A.; BISHOP, T.F.A.; DUNBAR, M.S. \& SHATAR, T.M. An overview of pedometric techniques for use in soil survey. Geoderma, 97:293-327, 2000 .

MIYASAKA, S. \& MEDINA, J.C. A soja no Brasil. Campinas, Instituto de Tecnologia de Alimentos, 1981. 1062p.

TOMLIN, D. Geographic information systems and cartographic modeling. New York, Prentice Hall, 1990. 362p.

UNIVERSIDADE FEDERAL DE SANTA MARIA - UFSM. Levantamento de reconhecimento dos solos do Estado de Santa Catarina. R. Centro de Ci. Rurais, 2:11-494, 1972.

VAUCLIN, M. Méthodes d'étude de la variabilité spatiale des propriétés d'un sol. In : Variabilité spatiale des processus de transfert dans les sols. Avignon, 1982. Paris, INRA Publ., p.9-43. 1983. (Les Colloques de l'INRA, $\mathrm{n}^{\circ} 15$ )

VIEIRA, S.D.Geoestatística em estudos de variabilidade espacial do solo. In: NOVAIS, R.F.; ALVAREZ V., V.H. \& SCHAEFER, C.E.G.R., eds. Tópicos em ciência do solo. v.1. Viçosa, Sociedade Brasileira de Ciência do Solo, 2000 . p.154 .

WEBSTER, R. The development of pedometrics. Geoderma, 62:115, 1994.

WEBSTER, R. Quantitative spatial analysis of soil in the field. Advances in Soil Science. New York, Springer Verlag, 1985. p.1-70. 